\title{
ON THE USE OF INVARIANCE REQUIREMENTS FOR INTERMEDIATE CONFIGURATIONS ASSOCIATED WITH THE POLAR DECOMPOSITION OF A DEFORMATION GRADIENT*
}

\author{
BY \\ J. CASEY (University of Houston) \\ AND \\ P. M. NAGHDI (University of California, Berkeley)
}

1. Introduction. In the description of the behavior of certain media, such as elastic-plastic and viscoelastic materials for example, it is sometimes useful to explicitly employ an intermediate configuration. It then becomes necessary to state invariance requirements in order to ensure that the effect of the intermediate configuration appears in constitutive relations in a physically meaningful way. With special reference to elastic-plastic materials, Green and Naghdi [1] proposed that independent invariance requirements be enforced for the intermediate stress-free configurations of plasticity. As emphasized by Casey and Naghdi [2,3], the invariance requirements of [1] are based on the idea that all configurations, whether global or local, which differ from any physically possible configuration of a continuum by a rigid displacement are physically indistinguishable. All such configurations should therefore play an equal role in any theory of material behavior. The invariance requirements guarantee that they do.

Now, for any continuum, a polar decomposition of the deformation gradient can always result in two intermediate local configurations, one through the right and the other through the left decomposition. The following question immediately arises: If invariance requirements are enforced for the intermediate configurations associated with the polar decomposition, are the results consistent with those derived in the usual manner, i.e., without any explicit use of these intermediate configurations? The purpose of this note is to show that the results are indeed consistent. This conclusion lends further justification for the use of the invariance requirements proposed in [1].

\footnotetext{
* Received November 5, 1982.

The results reported here were obtained in the course of research supported by the U. S. Office of Naval Research under Contract N00014-75-C0148, Project NR 064-436, with the University of California, Berkeley.
} 
2. Invariance requirements for intermediate configurations. Let a body be deformed from a fixed reference configuration $\kappa_{0}$ into any configuration $\kappa_{1}$, and subsequently from $\kappa_{1}$ into the present configuration $\kappa$. We recall from $[2,3]$ that the deformation gradient $\mathbf{F}$ in the configuration $\kappa$ (relative to $\kappa_{0}$ ) can always be expressed in product form as

$$
\mathbf{F}=\mathbf{F}_{2} \mathbf{F}_{1} \text {, }
$$

where $\mathbf{F}_{1}$ is the deformation gradient in $\kappa_{1}$ relative to $\kappa_{0}$ and $\mathbf{F}_{2}$ is the gradient in $\kappa$ relative to $\kappa_{1}$. All three tensors in (1) have positive determinants. If $\kappa$ is replaced by a configuration $\kappa^{+}$that differs from $\kappa$ by an arbitrary finite rigid displacement whose rotation tensor is $\mathbf{Q}$, then $\mathbf{F}$ is transformed into

$$
\mathbf{F}^{+}=\mathbf{Q F} \text {. }
$$

Similarly, if $\kappa_{1}$ is replaced by a configuration $\kappa_{1}^{+}$that differs from $\kappa_{1}$ by an arbitrary rigid displacement whose rotation tensor is $\overline{\mathbf{Q}}$, then $\mathbf{F}_{1}$ is transformed into

$$
F_{1}^{+}=\overline{\mathbf{Q}} \mathbf{F}_{1}
$$

and hence with the help of (1) and (2) we also have

$$
\mathbf{F}_{2}^{+}=\mathbf{F}^{+}\left(\mathbf{F}_{1}^{+}\right)^{-1}=\mathbf{Q} \mathbf{F}_{2} \overline{\mathbf{Q}}^{T} \text {. }
$$

Let the strain energy density $\psi$ in the configuration $\kappa$ be prescribed by a constitutive equation of the form

$$
\psi=\hat{\psi}\left(\mathbf{F}_{1}, \mathbf{F}_{2}\right)
$$

Then, the "full invariance requirements" of $[1,2,3]$ imply that $\psi$ is unaltered under the transformations (3) and (4) so that

$$
\hat{\psi}\left(\mathbf{F}_{1}, \mathbf{F}_{2}\right)=\hat{\psi}\left(\overline{\mathbf{Q}} \mathbf{F}_{1}, \mathbf{Q} \mathbf{F}_{2} \overline{\mathbf{Q}}^{T}\right)
$$

for all proper orthogonal $\mathbf{Q}, \overline{\mathbf{Q}}$. Thus, once an intermediate configuration, whether global or local, is explicitly employed, two (generally unequal) proper orthogonal tensors $\mathbf{Q}, \overline{\mathbf{Q}}$ appear in the invariance requirements.

3. Usual method of reducing constitutive equations. For the sake of clarity, we now briefly recall the main steps of the usual method of effecting the reduction of the constitutive response functions. To this end, it will suffice to consider an elastic material for which

$$
\psi=\bar{\psi}(\mathbf{F}) \text {. }
$$

The usual invariance requirement that $\psi$ be unaltered under the transformation (2) implies that

$$
\bar{\psi}(\mathbf{F})=\bar{\psi}(\mathbf{Q F})
$$

for all proper orthogonal $\mathbf{Q}$. Using a right polar decomposition of $\mathbf{F}$, we write

$$
\mathbf{F}=\mathbf{R} \mathbf{U}
$$

where the tensors $\mathbf{R}$ and $\mathbf{U}$ are proper orthogonal and symmetric positive definite, 
respectively. Next, we choose $\mathbf{Q}=\mathbf{R}^{T}$ in (8) and obtain

$$
\bar{\psi}(\mathbf{F})=\bar{\psi}(\mathbf{U}),
$$

which shows that the strain energy can depend on $\mathbf{F}$ only through $\mathbf{U}$. Besides being a necessary condition for (8), (10) is also sufficient. To see the latter, let $\mathbf{F}^{+}$be expressed in the polar form

$$
\mathbf{F}^{+}=\mathbf{R}^{+} \mathbf{U}^{+}
$$

From (2), (9) and (11) it is clear that

$$
\mathbf{U}^{+}=\mathbf{U}, \quad \mathbf{R}^{+}=\mathbf{Q R} .
$$

The result follows from (10) with the use of (12)।.

4. A special use of the invariance requirement (6). Returning to our main objective, in view of (1) and (9), we may choose

$$
\mathbf{F}_{1}=\mathbf{U}, \quad \mathbf{F}_{2}=\mathbf{R} .
$$

Thus, the intermediate configuration is now a local configuration that differs from the reference configuration by a pure stretch. Now, since $\mathbf{R}$ and $\mathbf{U}$ are uniquely determined by $\mathbf{F}$ (and vice versa), the constitutive assumption (7) can be written in the equivalent form

$$
\psi=\hat{\psi}(\mathbf{U}, \mathbf{R})
$$

which is of the same type as (5). Does the invariance requirement (6) then imply that (14) is dependent on $\mathbf{U}$ only, as the usual method does in (10)?

To pursue this question, we first decompose $\mathbf{F}_{1}$ and $\mathbf{F}_{2}$ in polar form

$$
\mathbf{F}_{1}=\mathbf{R}_{1} \mathbf{U}_{1}, \quad \mathbf{F}_{2}=\mathbf{R}_{2} \mathbf{U}_{2}
$$

and utilize (3) and (4) to obtain the transformation laws

$$
\begin{array}{ll}
\mathbf{U}_{1}^{+}=\mathbf{U}_{1}, & \mathbf{R}_{1}^{+}=\overline{\mathbf{Q}} \mathbf{R}_{1}, \\
\mathbf{U}_{2}^{+}=\overline{\mathbf{Q}} \mathbf{U}_{2} \overline{\mathbf{Q}}^{T}, & \mathbf{R}_{2}^{+}=\mathbf{Q} \mathbf{R}_{2} \overline{\mathbf{Q}}^{T} .
\end{array}
$$

Recalling the identifications (13), it follows from (15) and (16)that

$$
\begin{gathered}
\mathbf{R}_{1}=\mathbf{I}, \quad \mathbf{U}_{1}=\mathbf{U}, \quad \mathbf{R}_{2}=\mathbf{R}, \quad \mathbf{U}_{2}=\mathbf{I}, \\
\mathbf{U}_{1}^{+}=\mathbf{U}, \quad \mathbf{R}_{1}^{+}=\overline{\mathbf{Q}}, \quad \mathbf{F}_{1}^{+}=\overline{\mathbf{Q}} \mathbf{U}, \\
\mathbf{U}_{2}^{+}=\mathbf{I}, \quad \mathbf{F}_{2}^{+}=\mathbf{R}_{2}^{+}=\mathbf{Q R} \overline{\mathbf{Q}}^{T},
\end{gathered}
$$

where $\mathbf{I}$ is the identity tensor. It is important to note that while $\mathbf{F}_{1}$ is a pure stretch, in view of $(17)_{7}, \mathbf{F}_{1}^{+}$is generally not so.'

Returning to the reduction of the constitutive equation (14), it is easy to show that (10) is a necessary condition for satisfication of the invariance requirement (6) subject to the

\footnotetext{
'In this connection, see the discussion in [3] in regard to the inappropriateness of specifying that certain configurations in plasticity involve stretch only.
} 
identifications (13). Thus, the substitution of (13) in (6) leads to the condition that

$$
\hat{\psi}(\mathbf{U}, \mathbf{R})=\hat{\psi}\left(\overline{\mathbf{Q}} \mathbf{U}, \mathbf{Q R} \overline{\mathbf{Q}}^{T}\right)
$$

for all proper orthogonal $\mathbf{Q}, \overline{\mathbf{Q}}$. Choosing $\overline{\mathbf{Q}}=\mathbf{I}$ and $\mathbf{Q}=\mathbf{R}^{T}$, it follows from (18) that

$$
\hat{\psi}(\mathbf{U}, \mathbf{R})=\hat{\psi}(\mathbf{U}, \mathbf{I}) \text {, }
$$

which is equivalent to (10), since in view of (7) and (14)

$$
\hat{\psi}(\mathbf{U}, \mathbf{I})=\bar{\psi}(\mathbf{U}) \text {. }
$$

The proof of sufficiency is immediate, since although $\mathbf{F}_{1}^{+}$is given by $(17)_{7}, \mathbf{U}^{+}$satisfies $(12)_{1}$.

The intermediate configuration given by the left polar decomposition of the deformation gradient $\mathbf{F}$ may be treated in a similar manner.

\section{REFERENCES}

[1] A. E. Green and P. M. Naghdi, Some remarks on elastic-plastic deformation at finite strain, Int. J. Engng. Sci. $91219-1229(1971)$

[2] J. Casey and P. M. Naghdi, A remark on the use of the decomposition $\mathbf{F}=\mathbf{F}_{e} \mathbf{F}_{p}$ in plasticity, J. Appl. Mech. 47 $672-675(1980)$

[3] J. Casey and P. M. Naghdi, Discussion of "A correct definition of elastic and plastic deformation and its computational significance." J. Appl. Mech. 48 983-984 (1981) 